\title{
Studies of Some Naturally Occurring Auxotrophs of Neisseria gonorrhoeae
}

\author{
By ELLIOT JUNI* AND GLORIA A. HEYM \\ Department of Microbiology, The University of Michigan, \\ Ann Arbor, Michigan 48109, U.S.A.
}

(Received 11 March 1980)

\begin{abstract}
A strain of Neisseria gonorrhoeae requiring arginine, proline, glutamate and cystine as nutritional supplements was transformed, in several steps, to grow in a simple mineral medium containing cystine as the only growth factor with DNA from several clinically isolated strains of this organism. Using DNA from naturally occurring auxotrophs (auxotypes) known to require arginine, hypoxanthine and uracil ( $\mathrm{Arg}^{-} \mathrm{Hyx}^{-} \mathrm{Ura}^{-}$), as well as other factors, it was possible to transfer nutritional markers, one at a time, into such prototrophs to obtain seven single marker auxotrophic strains. Three different uracil markers, two different hypoxanthine markers, an arginine marker, and an isoleucine-valine marker were each introduced into separate strains. Of 114 DNA samples from independently isolated strains of $N$. gonorrhoeae, 54 were able to transform all seven single marker strains to prototrophy. Six of the single marker strains failed to be transformed to prototrophy by DNA samples from 43 strains, thus demonstrating that all these strains possess at least six nutritional lesions in common. Two strains were shown to contain all seven nutritional lesions, whereas several strains contained some but not all of the seven lesions. Six of the seven single marker strains have been shown to revert spontaneously to prototrophy at low frequencies. During construction of prototrophic strains it was observed that genes conferring sensitivity to growth inhibition by nutrients in complex media were occasionally transferred along with prototrophy.
\end{abstract}

\section{INTRODUCTION}

During an investigation of the use of genetic transformation as a tool for identification of strains of Neisseria gonorrhoeae it was shown that a series of proline auxotrophs of a particular strain of $N$. gonorrhoeae were transformed readily to prototrophy by DNA from other species of Neisseria (Janik et al., 1976). One uracil- and arginine-requiring auxotroph of this strain, however, was relatively much more specific for transformation with DNA from strains of $N$. gonorrhoeae; DNA from $N$. meningitidis and other neisserias was virtually inactive (Janik et al., 1976). The present investigation originated while attempting to obtain a large number of nutritional markers in $N$. gonorrhoeae in order to test these for possible specificity in interspecies transformation.

Because ultraviolet irradiation as well as most chemical mutagens appear to be relatively ineffective in inducing mutations in neisserias (Jyssum, 1965; Lie, $1965 a, c$ ), and also because the penicillin technique for selection of auxotrophs in a mutagenized population was not successful for these organisms (Lie, 1965b; E. Juni \& G. A. Heym, unpublished results), an attempt was made to study some of the many naturally occurring mutations in isolated strains of N. gonorrhoeae (Carifo \& Catlin, 1973; Catlin, 1973). Examination of a series of independently isolated strains of $N$. gonorrhoeae, all of which require at least arginine, hypoxanthine and uracil in order to grow in defined media, revealed that there are a group 
Table 1. Defined growth media

\begin{tabular}{|c|c|c|c|}
\hline \multirow[b]{2}{*}{ Component $\left(\mathrm{g} \mathrm{1}^{-1}\right)$} & \multicolumn{3}{|c|}{ Medium } \\
\hline & M9* & M52 & M55 \\
\hline Glucose $(3 \cdot 5)$ & + & + & + \\
\hline Sodium lactate $(2 \cdot 6)$ & + & + & + \\
\hline Mineral salts $\dagger$ & + & + & + \\
\hline Sodium glutamate $(0.22)$ & - & + & + \\
\hline Proline $(0.022)$ & - & - & + \\
\hline Arginine $(0.022)$ & - & - & + \\
\hline Cystine $(0.088)$ & - & + & + \\
\hline Hypoxanthine $(0.011)$ & - & - & + \\
\hline Casein hydrolysate $\ddagger(7 \cdot 0)$ & + & - & - \\
\hline Corn starch $\S(1 \cdot 3)$ & + & + & + \\
\hline Agar $\|(13 \cdot 0)$ & + & + & + \\
\hline
\end{tabular}

* Medium M9 has been used in a previous study (Janik et al., 1976).

$\uparrow$ The following mineral salts were used $\left(\mathrm{g} \mathrm{l}^{-1}\right) \mathrm{Na}_{2} \mathrm{HPO}_{4}, 2 \cdot 45 ; \mathrm{K}_{2} \mathrm{HPO}_{4}, 0.88 ; \mathrm{MgSO}_{4}, 0.2 ; \mathrm{NaCl}, 4.4$. $\ddagger$ Vitamin-free, salt-free casein hydrolysate (ICN Pharmaceuticals).

$\S$ Added as $88 \mathrm{ml}$ of an autoclaved $1.5 \%$ (w/v) cornstarch (Argo) solution per litre, in order to complex inhibitory fatty acids (Ley \& Mueller, 1946).

|l Difco.

of several markers each of which occurs in such strains. The present investigation explores the distribution of this group of nutritional markers in a collection of 114 clinically isolated strains of $N$. gonorrhoeae.

\section{METHODS}

Bacterial strains. The strains of $N$. gonorrhoeae examined were obtained from the diagnostic laboratory of St Joseph Mercy Hospital, Ann Arbor, Michigan, U.S.A., from A. Janik (Elkhart, Indiana, U.S.A.) and from the American Type Culture Collection. Strain 55, the recipient in most of the transformation studies described, was used in previous investigations (Bawdon et al., 1977; Janik et al., 1976).

Transformation procedures. The preparation of crude transforming DNA and procedures for transformation of nutritional markers have been described previously (Janik et al., 1976). Recipient strains of $N$. gonorrhoeae were maintained as colony types 1 or 2 using the method of Juni \& Heym (1977). Nutritional markers from auxotrophic strains were introduced into suitable strains by congression(Erickson \& Copeland, 1973; Nester et al., 1963) with either a streptomycin resistance marker or with another single nutritional marker for which the recipient strain was defective. In some cases the recipient strain, after exposure to DNA from an auxotrophic strain, was streaked for colony isolation on complex medium M1 (Janik et al., 1976) and individual colonies were assayed for transfer of the desired nutritional marker.

A series of strains each containing a single nutritional marker was used to assay for the presence or absence of the identical lesion in members of the collection of strains studied by testing DNA from each strain for ability to transform the recipient strain to prototrophy. DNA-recipient cell mixtures were streaked on any of the media listed in Table 1 that did not contain the required growth factor.

\section{RESULTS}

\section{Construction of prototrophic strains}

Strain 55 of $N$. gonorrhoeae grows well on medium M9 (Table 1). The vitamin-free casein hydrolysate in this medium could be replaced, for strain 55, by arginine, proline, glutamate and cystine (Table 1, medium M55). Although not absolutely required in medium M55 (Table 1), addition of hypoxanthine improved growth significantly.

Using DNA from other strains of $N$. gonorrhoeae it was possible to transform away the requirements for arginine and proline, one at a time. Such transformed prototrophs were able to grow on medium M52 (Table 1) which contains only glutamate and cystine as nutritional supplements. Glutamate serves as the nitrogen source in medium M52 and can be replaced by $\mathrm{NH}_{4} \mathrm{Cl}$. Cystine (or cysteine) is required for growth of all strains of 
Table 2. Transformation of single marker strains of $N$. gonorrhoeae

\begin{tabular}{|c|c|c|c|c|c|c|c|c|}
\hline \multirow[b]{2}{*}{ Class } & \multirow{2}{*}{$\begin{array}{l}\text { No. of } \\
\text { strains* }\end{array}$} & \multicolumn{7}{|c|}{ Single marker strains } \\
\hline & & Ura-162 & Ura-165 & Ura-488† & Hyx-143 & Нyx-165 & Arg-143‡ & Ilv-143 \\
\hline $\mathbf{A}$ & 54 & $+\S$ & + & + & + & + & + & + \\
\hline B & 43 & - & - & + & - & - & - & - \\
\hline $\mathbf{C}$ & 2 & - & - & - & - & - & - & - \\
\hline D & 1 & - & - & - & + & - & - & + \\
\hline $\mathrm{E}$ & 1 & - & - & + & + & - & - & + \\
\hline $\mathrm{F}$ & 9 & - & - & + & - & - & - & + \\
\hline$G$ & 2 & - & - & + & - & - & + & - \\
\hline $\mathbf{H}$ & 1 & + & + & + & - & - & - & - \\
\hline I & 1 & + & + & + & + & + & - & + \\
\hline
\end{tabular}

* Strains of $N$. gonorrhoeae.

$\uparrow$ The arginine requirement of Ura- 488 can be satisfied by citrulline but not by ornithine.

$¥$ Ornithine can replace arginine for growth of Arg-143.

$\S$ The symbol + indicates that DNA from each of the strains in the given class could transform the particular auxotroph to prototrophy, whereas the symbol - indicates that DNA from each of the strains in the given class failed to transform the particular auxotroph to prototrophy. In all cases where transformation of a single marker strain was observed there were 100 to 1000 prototrophic colonies on the sector streaked with the marker strain-DNA mixture. By contrast, except for rare revertants, no prototrophic colonies appeared when a negative result is reported.

$N$. gonorrhoeae (Catlin, 1973). Starting with two other strains of $N$. gonorrhoeae capable of growing on medium M9 containing hypoxanthine and uracil, it was possible to transform each of them, first to grow on medium M55 and then to grow on medium M52.

\section{Introduction of single nutritional markers into prototrophic strains}

Starting with a prototrophic strain, derived from strain 55 and able to grow on medium M52, single nutritional markers were introduced by transformation. DNA used for these studies was obtained from strains of $N$. gonorrhoeae which could grow on medium M9 but only if this medium was supplemented with hypoxanthine and uracil. A total of seven different nutritional markers (Table 2) were isolated in this way. Three different uracil markers, two different hypoxanthine markers, an arginine marker, and an isoleucine-valine marker were each separated from the multi-auxotrophic strains. It seems most likely that these constructed marker strains carry only a single lesion since each of them was transformed back to prototrophy with high efficiency using DNA from a prototrophic strain. The three uracil lesions were shown not to be identical since DNA from any one of the constructed uracil auxotrophs could transform the other uracil auxotrophs to prototrophy. A similar situation prevailed for the two constructed hypoxanthine auxotrophs.

One uracil marker, Ura-162, may be weakly linked to the high level streptomycin resistance marker since repeated examination of streptomycin-resistant transformants of a prototrophic strain, using DNA from a streptomycin-resistant derivative of one of the multiauxotrophic strains employed in this study, resulted in cotransformation of this particular marker in approximately one out of 100 streptomycin-resistant transformants. No other evidence for linkage of the markers was obtained in the congression experiments since appearance of the unselected markers occurred rarely, generally less than one in 200 colonies transformed for the selected marker.

Unlike Hyx-143, Hyx-165 (Table 2) is slightly leaky when incubated on media lacking hypoxanthine, but it is, nevertheless, easy to distinguish the larger colonies of transformed cells from the minute colonies of this leaky mutant strain.

The Ura-488 auxotroph has a dual requirement for uracil and arginine. Transformation of Ura-488 to grow on medium M52 supplemented with either uracil or arginine resulted 
in transformants all of which were also able to grow on medium M52 alone. For this reason Ura-488 is most likely to be a single lesion that results in a non-functional carbamoyl phosphate synthetase, an enzyme involved in both pyrimidine and arginine biosynthesis in bacteria (Piérard, 1966).

\section{Interstrain transformation of single auxotrophic marker strains}

Starting with a collection of 114 independently isolated strains of $N$. gonorrhoeae, it was shown that none of the seven auxotrophic isolated markers were present in 54 of the strains since DNA from each of these strains (Table 2, class A) was able to transform to prototrophy every strain carrying one of the seven single markers. By contrast, DNA derived from each of 43 other strains was unable to transform six of the seven single marker strains. Thus, each of these 43 strains (Table 2, class B) has two uracil markers, two hypoxanthine markers, and single markers for arginine and isoleucine-valine. Strain ATCC 27633, a representative of Catlin's auxotype 16 (Carifo \& Catlin, 1973), is a member of class B (Table 2). The unusual aspect of these findings is that all 43 strains in class B have at least six identical nutritional markers.

Strains in class B could be transformed to uracil independence using DNA from a prototrophic strain. DNA from such uracil-independent strains could now transform all three single uracil marker strains listed in Table 2. Consistent results were obtained when strains from class B were transformed to hypoxanthine independence.

Only three of the 114 strains studied, those in classes C and D (Table 2), contained the Ura-488 marker. The two strains in class $C$ contain the six markers common to strains of class B as well as Ura-488. Organisms in classes D to $\mathrm{H}$ (Table 2) contain some, but not all, of the seven nutritional markers studied. Strains in class F, for example, lack the Ilv-143 lesion but are otherwise identical with the large number of strains in class $\mathrm{B}$. The single strain in class I, that contains only the Arg-143 lesion, is apparently unique since none of the other six markers were found to occur alone in this study. Strain ATCC 27630, a representative of Catlin's auxotype 9, which requires hypoxanthine and proline in order to grow in media used for gonococcal auxotyping (Carifo \& Catlin, 1973), was examined and found to contain a hypoxanthine lesion different from the two hypoxanthine markers listed in Table 2. DNA from this strain transformed all seven single marker strains to prototrophy.

Transformation of all single marker strains was repeated four to six times for each DNA sample tested with uniform results. In several cases internal control experiments were performed to test the competency of the single marker strains with the transformation procedures employed, especially when a particular DNA sample failed to transform these strains to prototrophy. Using DNA from streptomycin-resistant mutants of six $\mathrm{Arg}^{-} \mathrm{Hyx}^{-} \mathrm{Ura}^{-}$ strains that failed to transform a single marker strain to prototrophy, it was always demonstrated that the same recipient cell-DNA mixture gave rise to extensive transformation to streptomycin resistance.

With the exception of Ura-488, all single marker strains listed in Table 2 were transformed readily to prototrophy by DNA derived from several strains of $N$. meningitidis.

\section{Stability of single marker strains}

Six of the seven single marker strains (Table 2) occasionally reverted spontaneously to prototrophy on minimal plates heavily streaked. Only Ura-488 consistently failed to show spontaneous reversion to prototrophy.

\section{Transformation of nutrient-sensitive markers}

During these studies where strain 55 was transformed for independence from single nutritional requirements by successive transformations, a large number of DNA samples from different strains of $N$. gonorrhoeae were used. Although these transformants were all able to grow on medium M52 (Table 1), some of these prototrophic strains grew extremely 
poorly on complex media such as M1 (Janik et al., 1976) compared with growth of the untransformed parent strain 55 . It has been reported that growth of some naturally occurring strains of $N$. gonorrhoeae is inhibited by specific amino acids or other growth factors (Catlin, 1973; Morello et al., 1976; Wade \& Young, 1977). Some strains, for example, are inhibited by addition of growth factor quantities of adenine to medium M9 (E. Juni \& G. A. Heym, unpublished results). Inability of some of the prototrophically transformed strains to grow well on complex media indicates that during transformation to prototrophy other markers concerned with sensitivity to growth inhibition by specific nutrients were also transferred into the recipient strain. The particular nutrients to which these transformants were sensitive have not been examined in this study.

\section{DISCUSSION}

Naturally occurring strains of $N$. gonorrhoeae appear to have accumulated spontaneous mutations in genes coding for enzymes of a wide variety of biosynthetic pathways (Carifo \& Catlin, 1973; Catlin, 1973, 1977). Depending upon the specific combination of nutritional factors required for growth of particular strains on a defined medium it has been possible to classify at least 35 different auxotrophs, referred to as auxotypes (Carifo \& Catlin, 1973; Catlin, 1977). Using suitable DNA preparations from various strains of $N$. gonorrhoeae it was possible to correct any specific nutritional defect in a particular auxotype by transformation (Catlin, 1974), thus demonstrating that nutritional deficiencies in these organisms probably arose spontaneously by mutation, such defects being repairable by genetic recombination.

Several common kinds of auxotypes have requirements for arginine, hypoxanthine and uracil ( $\left.\mathrm{Arg}-\mathrm{Hyx}^{-} \mathrm{Ura}^{-}\right)$as well as for one or more other nutrients (Carifo \& Catlin, 1973; Catlin, 1977). In the present study we have demonstrated that virtually all such $\mathrm{Arg}^{-} \mathrm{Hyx}^{-}$ $\mathrm{Ura}^{-}$- strains have at least six different mutational defects in common. Using six $\mathrm{Arg}^{-} \mathrm{Hyx}^{-}$ Ura- $^{-}$strains of $N$. gonorrhoeae, Catlin (1974) showed that DNA from two other Arg$\mathrm{Hyx}^{-} \mathrm{Ura}^{-}$strains failed to transform the other six strains to either $\mathrm{Arg}^{+}, \mathrm{Hyx}^{+}$or $\mathrm{Ura}^{+}$. DNA from other gonococci, however, could transform each of the markers in these $\mathrm{Arg}^{-} \mathrm{Hyx}^{-} \mathrm{Ura}^{-}$strains to prototrophy (Catlin, 1974). Compared with the frequency of transformation of the $\mathrm{Arg}^{-}$marker, the frequencies of transformation of these strains to $\mathrm{Hyx}^{+}$or $\mathrm{Ura}^{+}$were extremely low, a fact that Catlin (1974) attributed to the possible inability of transformed cells to initiate growth in the absence of the originally required growth factor. In view of our findings that there are two uracil and two hypoxanthine markers in most $\mathrm{Arg}^{-} \mathrm{Hyx}^{-} \mathrm{Ura}^{-}$strains, the relatively low frequencies of transformation to $\mathrm{Hyx}^{+}$or $\mathrm{Ura}^{+}$would appear to result from the necessity of transforming two probably unlinked markers to obtain transformants with the desired phenotype.

In another study (Mayer et al., 1977), transformation of several $\mathrm{Arg}^{-} \mathrm{Hyx}^{-} \mathrm{Ura}^{-}$strains to Ura ${ }^{+}$occurred at a frequency significantly lower than the frequencies of transformation of other markers. In this same study, however, there was a high frequency of transformation to $\mathrm{Hyx}^{+}$. Since only two recipient strains were examined for transformation to $\mathrm{Hyx}^{+}$, it is possible that these strains may have contained only single $\mathrm{Hyx}^{-}$markers, as shown for strains in classes $\mathrm{D}$ and $\mathrm{E}$ (Table 2). Alternatively, it is possible that the leaky Hyx- marker (Hyx-165) may have been overlooked in this investigation since transformation of only Hyx-143 to $\mathrm{Hyx}^{+}$does give rise to colonies that grow on a medium devoid of hypoxanthine, although such colonies are considerably smaller than colonies of a strain in which both the Hyx-143 and the Hyx-165 lesions have been repaired.

The occurrence of each of six markers in such a large number of different strains of $N$. gonorrhoeae (class B, Table 2), some of which were derived from individuals residing in different cities, and the absence of all these markers in an even larger group of strains (class A, Table 2), raises questions concerning the origin of this group of mutations. It has been 
suggested that all $\mathrm{Arg}^{-} \mathrm{Hyx}^{-} \mathrm{Ura}^{-}$strains were derived from a single ancestor (Mayer et al., 1977). Such strains, however, have been shown to differ from each other since they possess other non-identical nutritional defects and have been classified into at least eight auxotypes (Carifo \& Catlin, 1973).

It is well known that certain particular chromosomal sites are subject to spontaneous mutation at unusually high frequencies, compared with the rate of spontaneous mutation at other sites in the same gene, and these have been referred to as mutational hot spots (Benzer, 1961). It is possible that most, if not all, auxotypic mutations arose at mutational hot spots since, in addition to the results presented in this report, it has already been demonstrated by interstrain transformation that naturally occurring proline lesions in a variety of strains fall into five classes (Mayer et al., 1977) whereas there are only approximately eight different arginine lesions in a large number of arginine-requiring strains (Short et al., 1977; Young et al., 1977).

A possible hypothesis to explain the occurrence of identical multiple lesions in many strains assumes that all strains of $N$. gonorrhoeae were originally prototrophic and that a mutator gene having specificity for a particular sequence of bases in chromosomal DNA arose by spontaneous mutation in some strains. All genes containing this particular base sequence would, in time, become mutagenized. Some of the mutagenized genes, but not all, could be expected to produce non-functional gene products. All strains having a defective gene due to activity of this mutator gene will have the identical lesion in the affected gene. Mutator genes have been shown to occur in Neisseria (Jyssum, 1968; Jyssum \& Jyssum, 1968). It is by no means certain, however, that naturally occurring nutritional lesions in $N$. gonorrhoeae are due to mutator genes and other hypotheses are possible. Consistent with the concept that $\mathrm{Arg}^{-} \mathrm{Hyx}^{-} \mathrm{Ura}^{-}$strains can arise by spontaneous mutation is the finding in the recent report by Kenyon (1978) that during passage of a proline-requiring strain of $N$. gonorrhoeae in the normal mouse, genetic defects were acquired in some cells such that a strain could be isolated with additional requirements for arginine and uracil as well as a partial requirement for hypoxanthine.

Only three strains, in classes C and D (Table 2), contain the Ura-488 marker. Although the Ura-488 marker was also obtained following treatment with methyl methanesulphonate (Janik et al., 1976), it is possible that the single mutant isolated may have arisen by spontaneous mutation. Perhaps Ura-488 also represents a mutational hot spot although it occurs relatively rarely. Since all the naturally occurring mutations examined in this study, with the exception of Ura-488, have been shown to undergo spontaneous reversion it may be that strains in classes $\mathrm{E}$ to $\mathrm{H}$ (Table 2) were derived by spontaneous reversion of single markers originally present in these strains which prior to such reversion would reside in class B (Table 2). The single strain in class I (Table 2), which contains only the Arg-143 marker, is apparently unique since none of the other six markers appear to occur alone, at least not in any member of the collection of strains examined. It seems possible that a mechanism for creation of mutational hot spots may play a role in the evolution of nutritional requirements in strains of $N$. gonorrhoeae.

The natural process that resulted in the appearance of at least six identical nutritional lesions in large numbers of strains of $N$. gonorrhoeae may also have affected other properties as well (Catlin, 1977; Kenyon, 1978). It has been demonstrated that Arg-Hyx-Ura- strains appear to be involved especially in disseminated gonococcal infection (Brooks et al., 1976; Knapp \& Holmes, 1975; Morello et al., 1976; Schoolnik et al., 1976). Organisms with these same nutritional requirements have also been shown to predominate in asymptomatic gonorrhoea in males (asymptomatic urethral infection; Crawford et al., 1977).

The present studies have demonstrated that it is possible to transfer naturally occurring mutations showing sensitivity to certain growth factors, as well as nutritional markers, into a nutrient-insensitive prototrophic strain. Previous genetic investigations of $N$. gonorrhoeae have involved the use of a variety of strains each possessing several different properties 
(Catlin, 1967, 1974; Mayer et al., 1977; Young et al., 1977). The ability to transfer markers into a single uncomplicated prototrophic strain should be useful for future genetic analysis of $N$. gonorrhoeae.

This investigation was supported by Public Health Service grant AI-10107 from the National Institute of Allergy and Infectious Diseases, National Institutes of Health.

\section{REFERENCES}

Bawdon, R. E., Juni, E. \& BRITT, E. M. (1977). Identification of Neisseria gonorrhoeae by genetic transformation: a clinical laboratory evaluation. Journal of Clinical Microbiology 5, 108-109.

BenZER, S. (1961). On the topography of the genetic fine structure. Proceedings of the National Academy of Sciences of the United States of America 47, 403-415.

Brooks, G. F., Israel, K. S. \& Peterson, B. H. (1976). Bactericidal and opsonic activity against Neisseria gonorrhoeae in sera from patients with disseminated gonococcal infection. Journal of Infectious Diseases 134, 450-462.

Carifo, K. \& Catlin, B. W. (1973). Neisseria gonorrhoeae auxotyping: differentiation of clinical isolates based on growth responses on chemically defined media. Applied Microbiology 26, 223-230.

CAtlin, B. W. (1967). Genetic studies of sulfadiazineresistant and methionine-requiring Neisseria isolated from clinical material. Journal of Bacteriology 94, 719-733.

Catlin, B. W. (1973). Nutritional profiles of Neisseria gonorrhoeae, Neisseria meningitidis, and Neisseria lactamica in chemically defined media and the use of growth requirements for gonococcal typing. Journal of Infectious Diseases 128, 178-194.

Catlin, B. W. (1974). Genetic transformation of biosynthetically defective Neisseria gonorrhoeae clinical isolates. Journal of Bacteriology 120, 203-209.

Catlin, B. W. (1977). Nutritional requirements and auxotyping. In The Gonococcus, ch. 5. Edited by R. B. Roberts. New York: John Wiley.

Crawford, G., KNapp, J. S., Hale, J. \& Holmes, K. K. (1977). Asymptomatic gonorrhea in men: caused by gonococci with unique nutritional requirements. Science 196, 1352-1353.

ERICKSON, R. J. \& Copeland, J. C. (1973). Congression of unlinked markers and genetic mapping in the transformation of Bacillus subtilis 168 . Genetics 73, 13-21.

JANIK, A., Juni, E. \& HeYM, G. A. (1976). Genetic transformation as a tool for detection of Neisseria gonorrhoeae. Journal of Clinical Microbiology 4, 71-81.

Juni, E. \& Heym, G. A. (1977). Simple method for distinguishing gonococcal colony types. Journal of Clinical Microbiology 6, 511-517.

JYSSUM, K. (1965). Isolation of auxotrophs from Neisseria meningitidis. Acta pathologica et microbiologica scandinavica 63, 435-444.

JYssum, K. (1968). Mutator factor in Neisseria meningitidis associated with increased sensitivity to ultraviolet light and defective transformation. Journal of Bacteriology 96, 165-172.
JysSUM, K. \& JysSUM, S. (1968). Isolation of variants with increased mutability from Neisseria meningitidis. Acta pathologica et microbiologica scandinavica 74, 93-100.

KENYON, J. E. (1978). Neisseria gonorrhoeae: acquisition of auxotrophy in the mouse. Current Microbiology 1, 253-265.

KNAPP, J. S. \& Hol Mes, K. K. (1975). Disseminated gonococcal infections caused by Neisseria gonorrhoeae with unique nutritional requirements. Journal of Infectious Diseases 132, 204-208.

LEY, H. L. \& Mueller, J. H. (1946). On the isolation from agar of an inhibitor for Neisseria gonorrhoeae. Journal of Bacteriology 52, 453-460.

LIE, S. (1965a). The inactivating and mutagenic effect of ultraviolet irradiation on Neisseria meningitidis. Acto! pathologica et microbiologica scandinavica 63, 456-468.

LIE, S. $(1965 b)$. On the effect of penicillin in the production of auxotrophic mutants of Neisseria meningitidis. Acta pathologica et microbiologica scandinavica 63, 609-614.

LIE, S. (1965c). Production of auxotrophic mutants of Neisseria meningitidis by nitrous acid. Acta pathologica et microbiologica scandinavica 63, 615-622.

Mayer, L. W., Schoolnik, G. K. \& Falkow, S. (1977). Genetic studies on Neisseria gonorrhoeae from disseminated gonococcal infections. Infection and Immunity 18, 165-172.

Morello, J. A., Lerner, S. A. \& Bohnhoff, M. (1976). Characteristics of atypical Neisseria gonorrhoeae from disseminated and localized infections. Infection and Immunity 13, 15101516.

Nester, E. W., Schafer, M. \& Lederberg, J. (1963). Gene linkage in DNA transfer: a cluster of genes concerned with aromatic biosynthesis in Bacillus subtilis. Genetics 48, 529-551.

PIÉRARD, A. (1966). Control of the activity of Escherichia coli carbamoyl phosphate synthetase by antagonistic allosteric effectors. Science 154, 1572-1573.

Schoolnik, G. T., Buchanan, T. M. \& Holmes, K. K. (1976). Gonococci causing disseminated gonococcal infections are resistant to the bactericidal action of normal human sera. Journal of Clinical Investigation 58, 1163-1173.

Short, H. B., Ploscowe, V. B., Weiss, J. A. \& YounG, F. E. (1977). Rapid method for auxotyping multiple strains of Neisseria gonorrhoeae. Journal of Clinica' Microbiology 6, 244-248.

WAdE, T. J. \& YounG, F. E. (1977). Effect of branched chain amino acid combinations on the growth of Neisseria gonorrhoeae. Abstracts of the 
Annual Meeting of the American Society for Microbiology, D43, p. 76.

Young, F. E., Ploscowe, V. \& Short, H. (1977).

The application of DNA-mediated transformation to elucidation of the pathobiology of Neisseria gonorrhoeae. In Modern Trends in Bacterial Transformation and Transfection, pp. 307-320. Edited by A. Portolés, R. Lopéz \& M. Espinosa. Amsterdam: North-Holland Publishing Co. 